\title{
Analysis of the $\mathrm{U}_{2}$-edge $\mathrm{X}$-ray absorption spectra in $\mathrm{UO}_{2}$ using molecular dynamics simulations
}

Dmitry Bocharova,b,*, Melanie Chollet ${ }^{\mathrm{a}}$, Matthias Krack ${ }^{\mathrm{a}}$, Johannes Bertsch $^{\mathrm{a}}$, Daniel Grolimund ${ }^{\mathrm{a}}$, Matthias Martin ${ }^{\mathrm{a}}$, Alexei Kuzmin ${ }^{\mathrm{b}}$, Juris Purans $^{\mathrm{b}}$, Eugene Kotomin ${ }^{\mathrm{b}}$

${ }^{a}$ Paul Scherrer Institute, CH-5232 Villigen PSI, Switzerland

${ }^{b}$ Institute of Solid State Physics, University of Latvia, LV-1063 Riga, Latvia

\begin{abstract}
Uranium $\mathrm{L}_{3}$-edge $\mathrm{X}$-ray absorption spectroscopy was used to study the atomic structure of uranium dioxide $\left(\mathrm{UO}_{2}\right)$. The extended X-ray absorption fine structure (EXAFS) was interpreted within the ab initio multiple-scattering approach combined with classical molecular dynamics to account for thermal disorder effects. Several force field models were validated, and the role of multiple-scattering contributions was evaluated.
\end{abstract}

Keywords: Uranium dioxide; X-ray absorption spectroscopy; EXAFS;

Molecular dynamics; CP2K

\section{Introduction}

Uranium dioxide $\left(\mathrm{UO}_{2}\right)$ is an important industrial material employed as a fuel in nuclear reactors. However, the performance of conventional $\mathrm{UO}_{2}$ fuel is limited by, amongst others, the phenomena of pellet-cladding interaction

\footnotetext{
*Corresponding author

Email addresses: bocharov@latnet.lv (Dmitry Bocharov), matthias.krack@psi.ch (Matthias Krack), a.kuzmin@cfi.lu.lv ( Alexei Kuzmin)
} 
(PCI), fuel swelling and fission gas release. The doping of $\mathrm{UO}_{2}$ with small amounts of chromium sesquioxide $\left(\mathrm{Cr}_{2} \mathrm{O}_{3}\right)$ is technically applied to obtain a larger average grain size after the fuel sintering process [1]. A change in grain structure results in a higher plasticity of the fuel material as well as the retention of fission gases inside the fuel matrix is improved due to a larger grain size. However, the detailed structure of the chromium-bearing precipitates and their interface with the host $\mathrm{UO}_{2}$ lattice is not yet completely explained.

The complexity of the problem requires the joint use of advanced experimental and theoretical methods, which complement each other. X-ray absorption spectroscopy is a natural choice in this case, since it is able to provide information on the local structure of the host $\mathrm{UO}_{2}$ matrix (Fig. 1) as well as on the dopant and requires only a small sample size that is beneficial in the case of irradiated material $[2,3]$.

It was shown recently that X-ray absorption spectra can be also successfully used to validate empirical $[4,5,6]$ or ab initio [7] theoretical models. The first approach, called MD-EXAFS method, is used in the present study to compare several available force field models $[8,9,10,11,12,13,14,15,16]$ using the $\mathrm{U} \mathrm{L}_{3}$-edge extended X-ray absorption fine structure (EXAFS) of pure and Cr-doped $\mathrm{UO}_{2}$. In this method [4] the configuration-averaged EXAFS signal is calculated from first-principles within the multiple-scattering approach $[17,18]$ for a set of atomic configurations obtained from classical molecular dynamics (MD) simulation performed at the experimental temperature. This allows for an accurate description of both static and thermal disorder, so that the resulting configuration-averaged EXAFS signal can be 


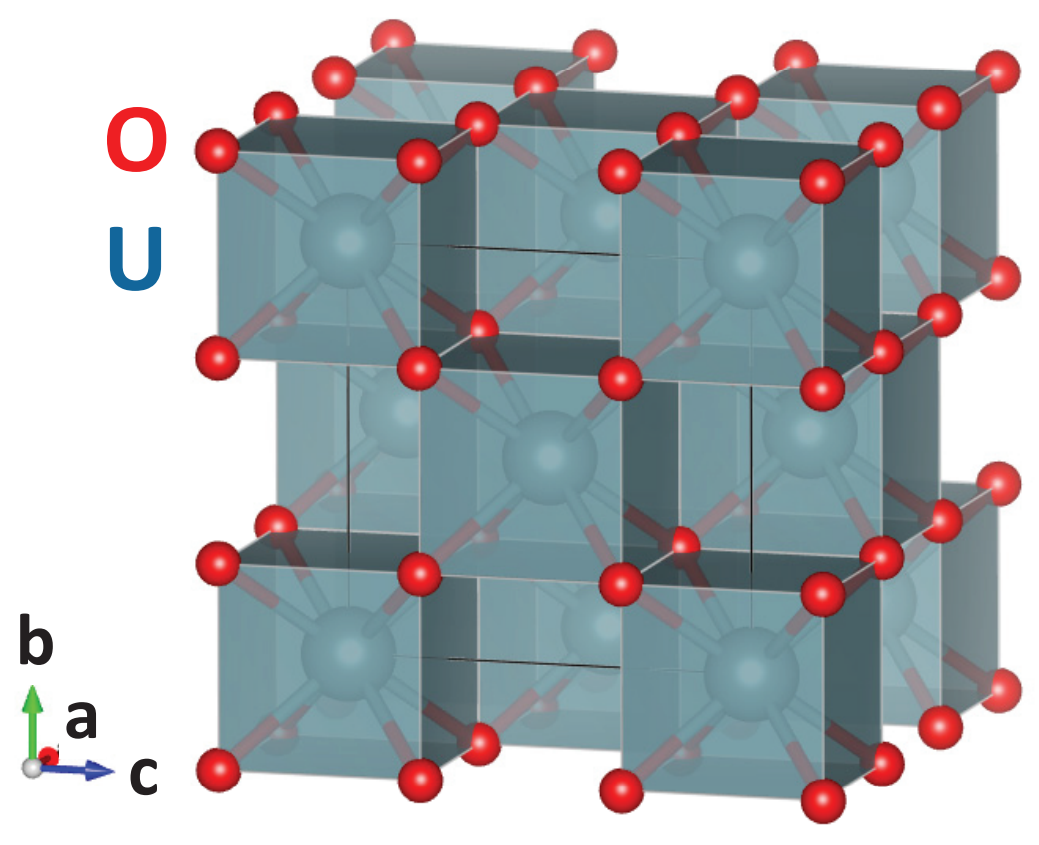

Figure 1: (Color online) Crystal structure of $\mathrm{UO}_{2}$ [19]. Large balls are uranium atoms, the small red balls are oxygen atoms.

directly compared with the experimental one. The residual between two signals is finally used to make a decision on the model quality.

\section{Experimental}

Two X-ray absorption spectroscopy experiments were performed on Crdoped and undoped $\mathrm{UO}_{2}$ samples at room temperature $(300 \mathrm{~K})$ using the radioactive microprobe endstation at the Swiss Light Source facility (Paul Scherrer Institute, Switzerland) microXAS beamline (undulator beam line X05LA [20]. The storage ring operated in the top-up mode at the energy $E=2.4 \mathrm{GeV}$ and current $I=400 \mathrm{~mA}$. The synchrotron radiation 
was monochromatized using a liquid nitrogen $\left(\mathrm{LN}_{2}\right)$ cryo-cooled, fixed-exit double-crystal Si(111) monochromator.

The monochromatic X-ray beam was focused to the focal spot size of approximately $2 \mu \mathrm{m}$ (horizontal) $\times 1 \mu \mathrm{m}$ (vertical) using an elliptical shape mirror pair, coated with Rh, in the Kirkpatrick-Baez (KB) geometry. The intensity of the incoming micro-beam before the sample was monitored by mini ion chamber (OHYO KOKEN KOGYO CO., LTD, Japan) continuously flushed with $\mathrm{N}_{2}$.

In the first experiment, an irradiated Cr-doped $\mathrm{UO}_{2}$ fuel pellet enriched with up to $4.8 \%{ }^{235} \mathrm{U}$ was used as a sample. The amount of $\mathrm{Cr}_{2} \mathrm{O}_{3}$ was 1600 ppm. Besides Cr a trace amount of aluminum was also present due to the use of Al-base lubricant within manufacturing step. The sample irradiation was performed in two cycles, with an average burnup of $\sim 40 \mathrm{MWd} / \mathrm{kg}$. The Cr K-edge (5989 eV) and $\mathrm{U} \mathrm{L}_{3}$-edge $(17166 \mathrm{eV})$ X-ray absorption spectra were collected in the fluorescence mode using an energy dispersive solid-state SDD (Si drift diode) detector. Additional experimental details can be found in $[2,3]$.

In the second experiment, we used non-irradiated undoped $\mathrm{UO}_{2}$ powder, manufactured at the Westinghouse plant (Vasteras, Sweden) ([21]). The powder was first pressed into green pellet and then sintered in a $\mathrm{H}_{2} / \mathrm{CO}_{2}$ gas mixture at $1800^{\circ} \mathrm{C}$ during 14 hours, leading to pellet density of about $10.60 \mathrm{~g} / \mathrm{cm}^{3}$. A disc with a thickness of $\sim 250 \mu \mathrm{m}$ was cut from the pellet, and a window of about $150 \times 150 \mu \mathrm{m}$ was thinned in the disc to $23 \mu \mathrm{m}$ by focused ion beam (FIB), to optimize sample thickness for X-ray transmission measurements. In the center of the fibbed window with uniform thickness 
Table 1: Description of the $\mathrm{UO}_{2}$ force fields used in the molecular dynamics simulations and calculated lattice constant $a_{0}$.

\begin{tabular}{|c|c|c|c|c|c|c|c|}
\hline & & Interaction & & Ion charges & & & \\
\hline Force field & $\mathrm{O}-\mathrm{O}$ & $\mathrm{O}-\mathrm{U}$ & $\mathrm{U}-\mathrm{U}$ & $\mathrm{Q}_{\mathrm{U}}$ & $\mathrm{QO}_{\mathrm{O}}$ & Shells & $a_{0}[\AA]$ \\
\hline Arima [8] & Buckingham & Buckingham & - & +4 & -2 & No & 5.4532 \\
\hline Basak [9] & Buckingham, Morse & Buckingham, Morse & Buckingham, Morse & +2.4 & -1.2 & No & 5.4547 \\
\hline Imperial [10] & Buckingham & Buckingham & - & +4 & -2 & Yes & 5.4677 \\
\hline Karakasidis [11] & Four-range Buckingham & Buckingham & - & +4 & -2 & No & 5.4657 \\
\hline Meis [12] & Four-range Buckingham & Buckingham & - & +4 & -2 & Yes & 5.4682 \\
\hline Morelon [13] & Four-range Buckingham & Buckingham & - & +3.227252 & -1.613626 & No & 5.4473 \\
\hline Read [14] & Four-range Buckingham & Buckingham & - & +4 & -2 & Yes & 5.4693 \\
\hline Yakub [16] & Buckingham & Buckingham, Morse & Buckingham & +2.2208 & -1.1104 & No & 5.4440 \\
\hline Yamada [8] & Buckingham, Morse & Buckingham, Morse & Buckingham, Morse & +2.4 & -1.2 & No & 5.4667 \\
\hline
\end{tabular}

$\mathrm{U} \mathrm{L}_{3}$-edge $\mathrm{X}$-ray absorption spectra were recorded at room temperature in transmission mode using an ionization chamber as detector.

Finally, we also used for comparison high-quality $\mathrm{U} \mathrm{L}_{3}$-edge X-ray absorption spectrum of $\mathrm{UO}_{2}$, measured in transmission mode at $300 \mathrm{~K}$ at $\mathrm{ROBL}$ BM-20 beamline at the ESRF synchrotron radiation facility [22].

\section{Classical Molecular Dynamics simulations}

Atomic scale simulations were carried out using the FisT module of the CP2K code [23]. Details about the implementation can be found in [24] and validation results are presented in [25]. The $\mathrm{UO}_{2}$ force fields of Arima et al. [8], Basak et al. [9], Grimes et al. (Imperial) [10], Karakasidis et al. [11], Meis et al. [12], Morelon et al. [13], Read et al. [14], Yamada et al. [15] and Yakub et al. [16] were employed to simulate uranium dioxide (see Table 1 for further details about these force fields).

The $\mathrm{UO}_{2}$ lattice constants obtained for all force fields after relaxation are 
also shown in Table 1 (last column). These results agree quite well with the experimental values $a_{0}=5.468(1) \AA[19]$ and 5.4731(4) $\AA[26]$. Only the force fields of Morelon [13] and Yakub [16] give slightly worse values.

The classical MD simulations were performed within the canonical (NVT) ensemble at $300 \mathrm{~K}$ using periodic boundary conditions for a $6 a_{0} \times 6 a_{0} \times 6 a_{0}$ supercell containing 2592 atoms with a lattice constant $a_{0}=5.4682 \AA$. All samples were carefully thermalized. Thereafter MD simulation runs of 20 ps using an MD time step interval of 0.5 fs were performed. A set of 4000 atomic configurations was retrieved from each MD simulation run.

The calculated radial distribution functions (RDFs) $G(r)$ for three selected force field models (Meis [12], Arima [8] and Imperial [10]) are shown in Fig. 2, and the values of the mean-square relative displacements (MSRDs) $\sigma^{2}$ obtained by all force field models for the first three coordination shells of uranium are given in Table 2.

\section{MD-EXAFS simulations}

The atomic configurations, obtained as a result of the classical NVT MD simulations for each force field model (Section 3) were used to generate configuration averaged EXAFS spectra which can be directly compared to the experimental data measured at the same temperature $[4,5]$. The averaging was performed over 4000 configurations to guarantee convergence.

For each atomic configuration, the EXAFS signal was calculated using the ab initio real-space multiple-scattering code FEFF9.64 [31, 17] taking into account all multiple-scattering paths within the cluster of $6 \AA$ radius around the absorbing uranium atoms. Thus all scattering events up to the $5^{\text {th }}$ order 
D. Bocharov, M. Chollet, M. Krack, J. Bertsch, D. Grolimund, M. Martin, A. Kuzmin, J. Purans, E. Kotomin, Analysis of the $\mathrm{U}$ L3-edge X-ray absorption spectra in $\mathrm{UO} 2$ using molecular dynamics simulations,

Prog. Nucl. Energy 94 (2017) 187-193.

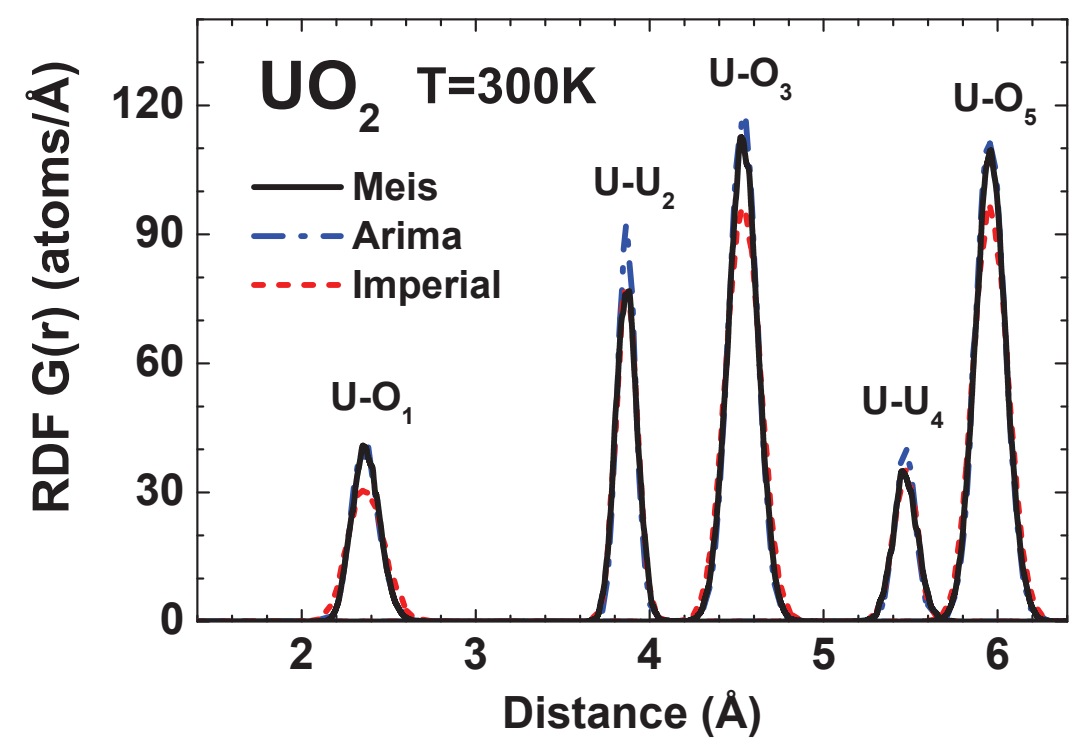

Figure 2: (Color online) Radial distribution functions (RDFs) $G(R)$ calculated for $\mathrm{UO}_{2}$ at $T=300 \mathrm{~K}$ using the rigid-ion potential of Arima et al. [8] and the core-shell potentials of Meis et al. [12] and Grimes et al. (Imperial) [10]. The first five coordination shells are indicated. 
D. Bocharov, M. Chollet, M. Krack, J. Bertsch, D. Grolimund, M. Martin, A. Kuzmin, J. Purans, E. Kotomin, Analysis of the $\mathrm{U}$ L3-edge X-ray absorption spectra in $\mathrm{UO} 2$ using molecular dynamics simulations, Prog. Nucl. Energy 94 (2017) 187-193.

Table 2: Calculated mean-square relative displacements (MSRDs) $\sigma^{2}$ for the first three coordination shells of uranium in $\mathrm{UO}_{2}$ at $T=300 \mathrm{~K}$. The experimental EXAFS results for irradiated Cr-doped $\mathrm{UO}_{2}$ from [3] and pure $\mathrm{UO}_{2}$ from [27], [28] and [29] at $T=300 \mathrm{~K}$ and from [30] at $T=200 \mathrm{~K}$ are given for comparison.

\begin{tabular}{lccc}
\hline & \multicolumn{3}{c}{$\mathrm{MSRD}^{2}\left(\AA^{2}\right)$} \\
& $\mathrm{U}-\mathrm{O}_{1}$ & $\mathrm{U}-\mathrm{U}_{2}$ & $\mathrm{U}-\mathrm{O}_{3}$ \\
\hline This work & 0.0060 & $\mathrm{EXAFS}$ \\
{$[3]$} & 0.004 & 0.006 \\
{$[27]$} & 0.009 & 0.005 \\
{$[28]$} & 0.0065 & 0.0055 & \\
{$[29]$} & 0.005 & & \\
{$[30]$} & 0.0086 & 0.0030 & \\
& & & \\
& & & \\
Meis & 0.0063 & 0.0038 & 0.0075 \\
Read & 0.0074 & 0.0042 & 0.0084 \\
Yakub & 0.0051 & 0.0043 & 0.0062 \\
Yamada & 0.0058 & 0.0039 & 0.0068 \\
& & & \\
Arima & 0.0066 & 0.0028 & 0.0067 \\
Basak & 0.0063 & 0.0043 & 0.0069 \\
Imperial & 0.0108 & 0.0039 & 0.0098 \\
Karakasidis & 0.0120 & 0.0037 & 0.0116 \\
Morelon & 0.0087 & 0.0041 & 0.0095 \\
\hline
\end{tabular}


were considered. The cluster radius was chosen to include five coordination shells giving dominant contributions into the experimental $\mathrm{U} \mathrm{L}_{3}$-edge EXAFS: (a) three (at $2.37 \AA, 4.53 \AA$ and $5.96 \AA$ ) composed of oxygen atoms and (b) two (at $3.87 \AA$ and $5.47 \AA$ ) composed of uranium atoms. The amplitude of the calculated EXAFS signals was scaled by a factor $S_{0}^{2}=0.83$, which was estimated from the prior analysis of the first shell $\mathrm{U}-\mathrm{O}_{1}$ EXAFS signal. The calculation of the cluster potential was performed only once for the average atom configuration, corresponding to the $\mathrm{UO}_{2}$ crystallographic structure [19]. The complex exchange-correlation Hedin-Lundqvist potential and default values for the muffin-tin radii $\left(R_{\mathrm{mt}}(\mathrm{U})=1.64 \AA\right.$ and $\left.R_{\mathrm{mt}}(\mathrm{O})=1.04 \AA\right)$, as provided within the FEFF code [31], were employed.

\section{Results and discussion}

The comparison of the total RDFs $G(r)$ (Fig. 2) and MSRD factors (Table 2) for the first three coordination shells of uranium allows one to draw several conclusions.

In the first coordination shell $\left(\mathrm{U}-\mathrm{O}_{1}\right)$ the smallest thermal disorder is predicted by the models of Yakub et al. [16] and Yamada et al. [15]. Slightly larger values are given by the models of Basak et al. [9], Meis [12] and Arima et al. [8], followed by the models of Read et al. [14] and Morelon et al. [13]. Finally, the $\operatorname{MSRD}\left(\mathrm{U}-\mathrm{O}_{1}\right)$ factors for the Imperial [10] and Karakasidis [11] model are nearly twice larger than our experimental result.

In the second coordination shell $\left(\mathrm{U}-\mathrm{U}_{2}\right)$, the scatter of the MSRD values is not as large as in the first shell. All models give close results around $0.004 \AA^{2}$, except for the model of Arima et al. [8], predicting much smaller 
disorder.

The thermal disorder in the third coordination shell $\left(\mathrm{U}-\mathrm{O}_{3}\right)$ correlates quite well with that in the first shell. The smallest $\operatorname{MSRD}\left(\mathrm{U}-\mathrm{O}_{3}\right)$ values are found for the models of Yakub et al. [16], Arima et al. [8], Yamada et al. [15], and Basak et al. [9]. Slightly larger values of MSRD are predicted by the models of Meis [12] and Read et al. [14], followed by the models of Morelon et al. [13], Imperial [10] and Karakasidis et al. [11].

The theoretical MSRD values for the $\mathrm{U}-\mathrm{O}_{1}, \mathrm{U}-\mathrm{U}_{2}$, and $\mathrm{U}-\mathrm{O}_{3}$ atom pairs agree reasonably well with the experimental ones obtained from the analysis of the $\mathrm{U} \mathrm{L}_{3}$-edge EXAFS data at $T=300 \mathrm{~K}$ in previous works [3, 29, 27, 28]. Note also that the MSRD values obtained at low temperature $T=200 \mathrm{~K}$ in [30] are also quite close, taking into account the dispersion of the published results.

Configuration-averaged $\mathrm{U} \mathrm{L}_{3}$-edge EXAFS spectrum $\chi(k) k^{2}$ produced for the Meis force field [12] is compared with the available experimental data in both $k$ - and $R$-spaces in Fig. 3. The same comparison for other force fields only in $R$-space is shown in Fig. 4 . Note that a comparison in the $R$-space is shown only for the longest experimental EXAFS from [22]. One can conclude that all nine force field models except Basak and especially Arima are able to reproduce the lattice dynamics in $\mathrm{UO}_{2}$ at $T=300 \mathrm{~K}$ rather well for outer coordination shells. However, the Morelon, Karakasidis and Imperial force field overestimate the thermal disorder in the first coordination shell. That results in the lower first peak amplitude in FTs at $\sim 1.9 \AA$.

The analysis of the coordination shells above the first one should account for the presence of multiple-scattering (MS) contributions correspond- 
D. Bocharov, M. Chollet, M. Krack, J. Bertsch, D. Grolimund, M. Martin, A. Kuzmin, J. Purans, E. Kotomin, Analysis of the $\mathrm{U}$ L3-edge X-ray absorption spectra in $\mathrm{UO} 2$ using molecular dynamics simulations, Prog. Nucl. Energy 94 (2017) 187-193.
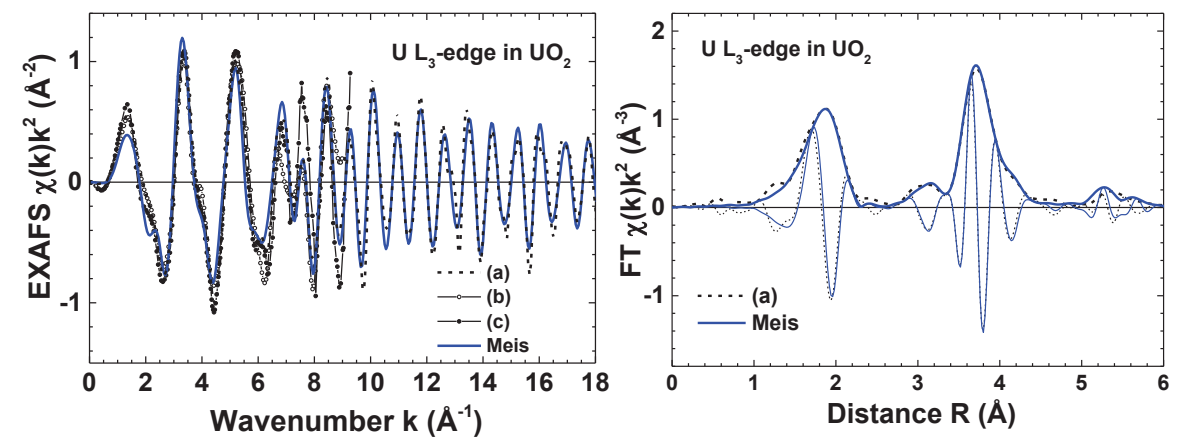

Figure 3: Comparison of the experimental and calculated $\mathrm{U} \mathrm{L}_{3}$-edge EXAFS $\chi(k) k^{2}$ and their Fourier transforms (FTs) (modulus and imaginary parts shown with thick and thin lines, correspondingly) for $\mathrm{UO}_{2}$ at $T=300 \mathrm{~K}$. The experimental EXAFS signals correspond to (a) $\mathrm{UO}_{2}$ from [22], (b) irradiated Cr-doped $\mathrm{UO}_{2}$ and (c) non-irradiated undoped $\mathrm{UO}_{2}$. The configuration-averaged EXAFS signal was calculated by the MD-EXAFS method at $T=300 \mathrm{~K}$ using the core-shell potential of Meis et al. [12]. The FTs were calculated employing $10 \%$-Gaussian window function in the $k$-space range $k=0.5-18.0 \AA^{-1}$. 
ing to many-atom distribution functions. These were estimated for the Meis force field model in Fig. 5, where separate contributions due to total single-scattering (SS), double-scattering (DS) and triple-scattering (TS) events are shown. Note, that these MS signals include both radial and angular thermal disorder effects properly accounted during the calculation of the configuration-averaged EXAFS. As one can see, the DS and TS contributions are present in $k$-space mainly below $7 \AA^{-1}$ and in $R$-space from $2.5 \AA$ to $5.5 \AA$, thus, affecting the outer shell signals, mainly under the second $\left(\mathrm{U}-\mathrm{U}_{2}\right)$ and third $\left(\mathrm{U}-\mathrm{O}_{3}\right)$ shells. The largest DS contribution comes from the scattering path $\mathrm{U}-\mathrm{O}_{1}^{\prime}-\mathrm{O}_{1}^{\prime \prime}-\mathrm{U}(\mathrm{DS} 1)$ (peak at $3 \AA$ ) and from a sum of scattering paths $\mathrm{U}-\mathrm{O}_{1}^{\prime}-\mathrm{O}_{1}^{\prime \prime \prime}-\mathrm{U}(\mathrm{DS} 2)$ and $\mathrm{U}-\mathrm{O}_{1}-\mathrm{U}_{2}-\mathrm{U}(\mathrm{DS} 3)$ (peak at $3.9 \AA$ ). The main TS contribution into the small peak at $4.2 \AA$ comes from the linear $\mathrm{O}-\mathrm{U}-\mathrm{O}$ scattering paths within the first coordination shell.

The analysis of MS effects allows us to conclude that while their contribution is not negligible in $\mathrm{UO}_{2}$, it is not so crucial as in the case of perovskitetype compounds $[32,33]$. Finally, one should also point out that the SS contribution from the second shell $\mathrm{U}-\mathrm{U}_{2}$ is responsible for a double-hump shape of the peak between $2.5 \AA$ and $4.5 \AA$ in the Fourier transform of the EXAFS (Fig. 5) due to the peculiar behaviour of the scattering amplitude of the heavy (uranium) atom [32].

\section{Conclusions}

Based on EXAFS spectra, a validation of $\mathrm{UO}_{2}$ force fields has been performed using the MD-EXAFS approach [4]. We have shown that the nine different $\mathrm{UO}_{2}$ force fields differ mainly in the description of the $\mathrm{U}-\mathrm{O}$ vi- 
D. Bocharov, M. Chollet, M. Krack, J. Bertsch, D. Grolimund, M. Martin, A. Kuzmin, J. Purans, E. Kotomin, Analysis of the $\mathrm{U}$ L3-edge X-ray absorption spectra in $\mathrm{UO} 2$ using molecular dynamics simulations, Prog. Nucl. Energy 94 (2017) 187-193.

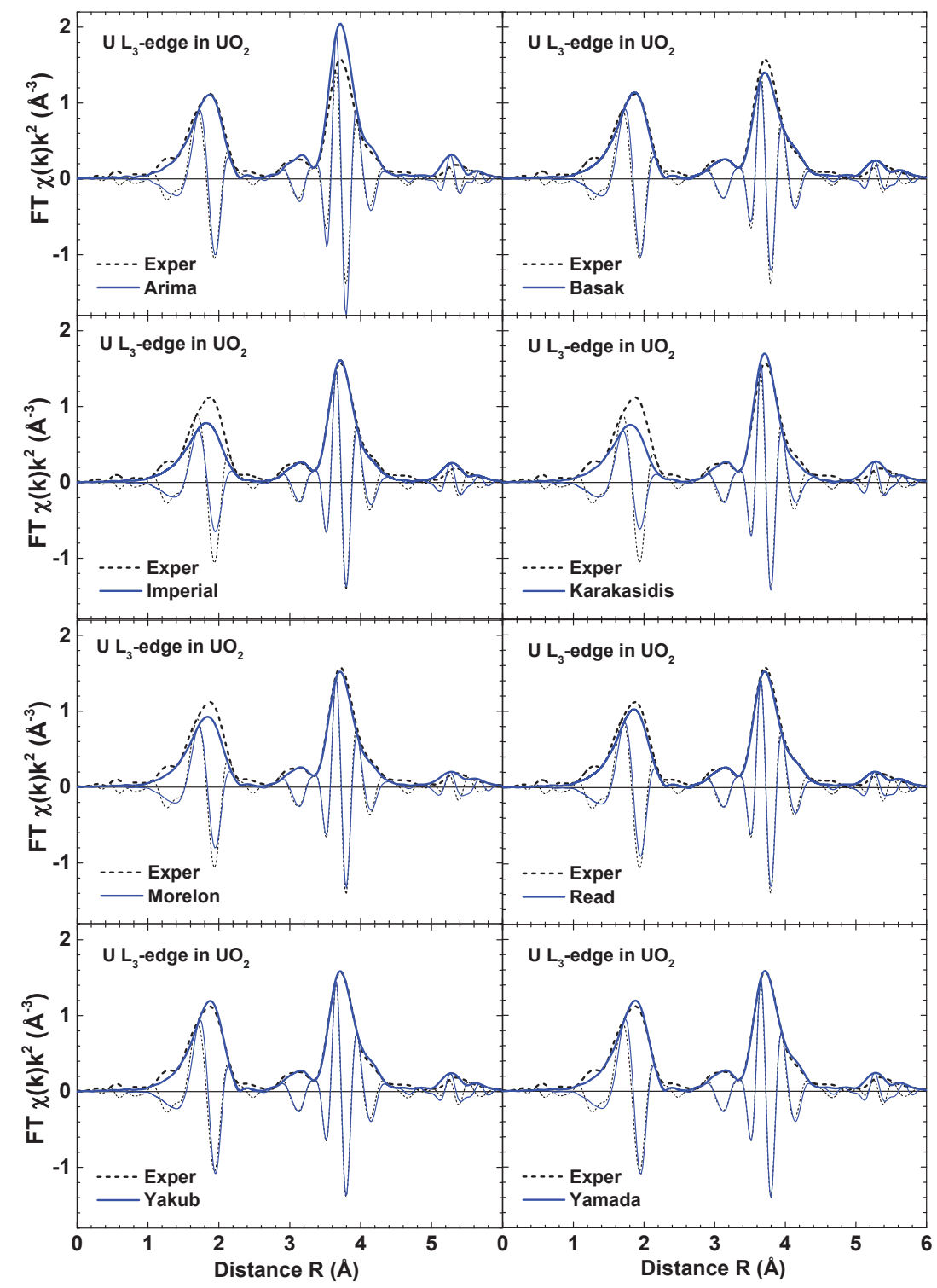

Figure 4: (Color online) Comparison of the Fourier transforms (FTs) (modulus and imaginary parts shown with thick and thin lines, correspondingly) of the experimental and calculated $\mathrm{U} \mathrm{L}_{3}$-edge EXAFS of $\mathrm{UO}_{2}$ at $T=300 \mathrm{~K}$. The experimental EXAFS signals correspond to (a) $\mathrm{UO}_{2}$ from [22]. The configuration-averaged EXAFS signal was calculated by the MD-EXAFS method at $T=300 \mathrm{~K}$ using eight different $\mathrm{UO}_{2}$ force fields from the literature $[8,9,10,11,13,14,15,16]$. The FTs were calculated employing $10 \%$-Gaussian window function in the $k$-space range $k=0.5-18.0 \AA^{-1}$. 
D. Bocharov, M. Chollet, M. Krack, J. Bertsch, D. Grolimund, M. Martin, A. Kuzmin, J. Purans, E. Kotomin, Analysis of the $\mathrm{U}$ L3-edge X-ray absorption spectra in $\mathrm{UO} 2$ using molecular dynamics simulations,

Prog. Nucl. Energy 94 (2017) 187-193.
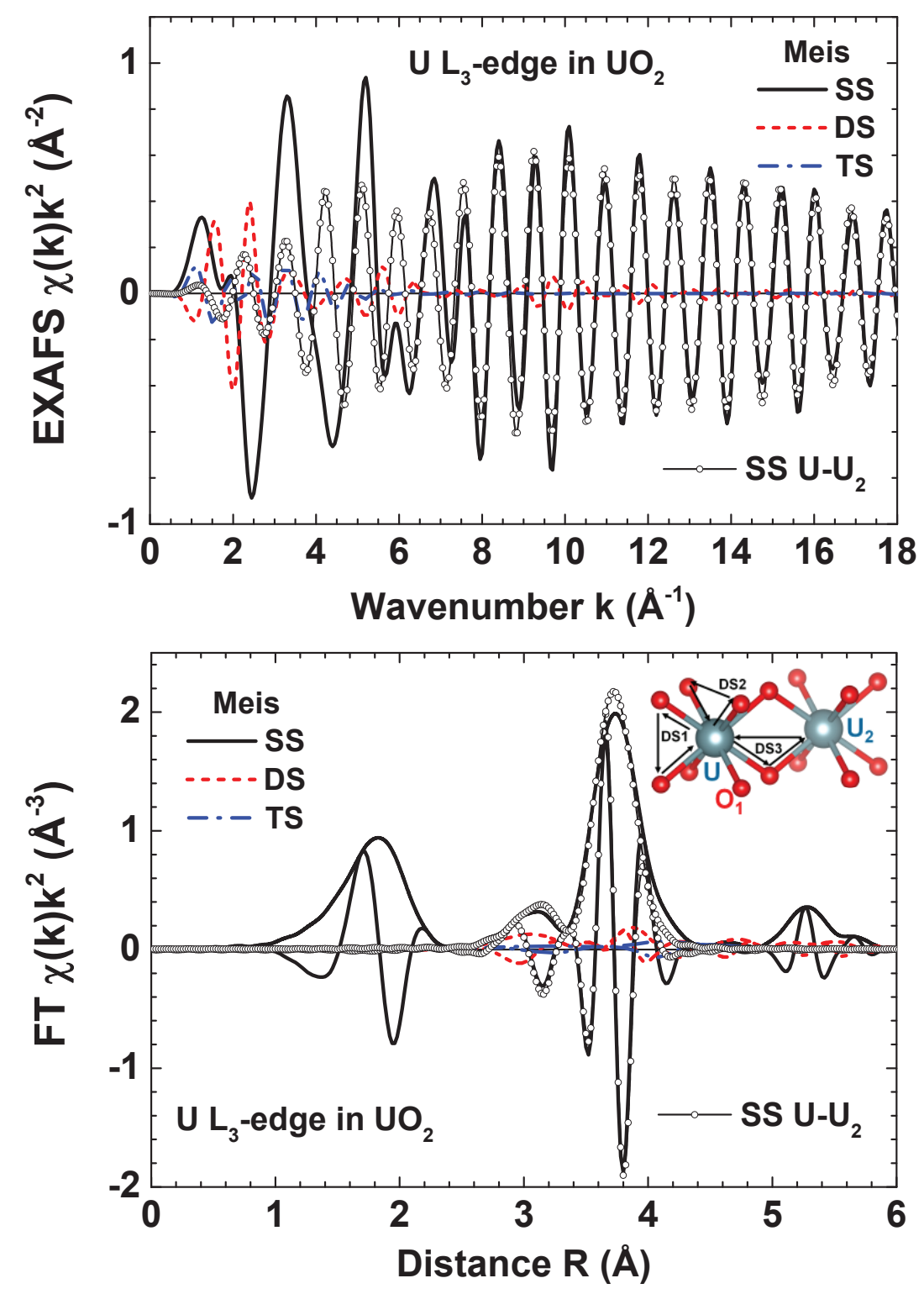

Figure 5: (Color online) Total single-scattering (SS), double-scattering (DS) and triplescattering (TS) contributions into the $\mathrm{U} \mathrm{L}_{3}$-edge MD-EXAFS spectrum $\chi(k) k^{2}$, obtained with the force field model from [12] at $T=300 \mathrm{~K}$, and their Fourier transforms (FTs) (both modules and imaginary parts are shown). The SS contribution due to the second coordination shell $\mathrm{U}-\mathrm{U}_{2}$ and the main DS paths are also shown. The FTs were calculated employing 10\%-Gaussian window function ip the $k$-space range $k=0.5-18.0 \AA^{-1}$. 
brational amplitudes. The best results for $\mathrm{UO}_{2}$ are obtained for the force field of Meis [12]. Basak, Read, Yamada, and Yakub show also a very good agreement. Morelon performs only slightly worse compared to the former ones, whereas Arima, Imperial, and Karakasides show clear deficiencies. The importance of the multiple-scattering contributions for EXAFS analysis was also evaluated. The obtained results suggest that they are important in the range of the second $\left(\mathrm{U}_{2}\right)$ and third $\left(\mathrm{O}_{3}\right)$ coordination shells of uranium.

\section{Acknowledgments}

This work was supported by a grant from the Swiss National Supercomputing Centre (CSCS) under the project ID s444. The resource allocation within the PSI share at CSCS and on the PSI compute cluster Merlin4 is also acknowledged. D. B. is grateful for a fellowship within the Sciex-NMS programme. A. K. was supported by Latvian Science Council Grant no. $187 / 2012$.

\section{References}

[1] A. Leenaers, L. de Tollenaere, C. Delafoy, S. V. den Berghe, J. Nucl. Mater. 317 (2003) 62-68.

[2] G. Kuri, C. Mieszczynski, M. Martin, J. Bertsch, C. Borca, C. Delafoy, J. Nucl. Mater. 449 (2014) 158-167.

[3] C. Mieszczynski, G. Kuri, J. Bertsch, M. Martin, C. N. Borca, C. Delafoy, E. Simoni, J. Phys.: Condens. Matter 26 (2014) 355009. 
[4] A. Kuzmin, R. A. Evarestov, J. Phys: Condens. Matter 21 (2009) 055401.

[5] A. Kuzmin, R. A. Evarestov, J. Phys.: Conf. Series 190 (2009) 012024.

[6] J. Timoshenko, A. Anspoks, A. Kalinko, A. Kuzmin, Acta Mater. 79 (2014) 194-202.

[7] E. Blokhin, E. Kotomin, A. Kuzmin, J. Purans, R. Evarestov, J. Maier, Appl. Phys. Lett. 102 (2013) 112913.

[8] T. Arima, S. Yamasaki, Y. Inagaki, K. Idemitsu, J. Alloys Compd. 400 (2005) 43-50.

[9] C. B. Basak, A. K. Sengupta, H. S. Kamath, J. Alloys Compd. 360 (2003) 210-216.

[10] R. W. Grimes, C. R. A. Catlow, Phil. Trans. R. Soc. Lond. A 335 (1991) 609-634.

[11] T. Karakasidis, P. J. D. Lindan, J. Phys.: Condens. Matter 6 (1994) 2965-2969.

[12] C. Meis, A. Chartier, J. Nucl. Mater. 341 (2005) 25-30.

[13] N. D. Morelon, D. Ghaleb, J. M. Delaye, L. Van Brutzel, Philos. Mag. 83 (2003) 1533-1555.

[14] M. S. Read, R. A. Jackson, J. Nucl. Mater. 406 (2010) 293 - 303.

[15] K. Yamada, K. Kurosaki, M. Uno, S. Yamanaka, J. Alloys Compd. 307 (2000) 10-16. 
[16] E. Yakub, C. Ronchi, D. Staicu, J. Nucl. Mater. 389 (2009) 119-126.

[17] J. J. Rehr, R. C. Albers, Rev. Mod. Phys. 72 (2000) 621-654.

[18] J. J. Rehr, J. J. Kas, M. P. Prange, A. P. Sorini, Y. Takimoto, F. Vila, C. R. Physique 10 (2009) 548-559.

[19] L. Desgranges, G. Baldinozzi, G. Rousseau, J.-C. Nipce, G. Calvarin, Inorg. Chem. 48 (2009) 7585-7592.

[20] C. N. Borca, D. Grolimund, M. Willimann, B. Meyer, K. Jefimovs, J. Vila-Comamala, C. David, J. Phys.: Conf. Series 186 (2009) 012003.

[21] J. Arborelius, K. Backman, L. Hallstadius, M. Limbäck, J. Nilsson, B. Rebensdorff, G. Zhou, K. Kitano, R. Löfström, G. Rönnberg, J. Nucl. Sci. \& Technol. 43 (2006) 967-976.

[22] G. Heisbourg, S. Hubert, N. Dacheux, J. Purans, J. Nucl. Mater. 335 (2004) 5-13.

[23] C. developers group, (http://www.cp2k.org), 2000-2015.

[24] F. Devynck, M. Iannuzzi, M. Krack, Phys. Rev. B 85 (2012) 184103.

[25] M. Krack, Mater. Res. Soc. Symp. Proc. 1383 (2012) mrsf11-1383-a0311.

[26] M. Idiri, T. L. Bihan, S. Heathman, J. Rebizant, Phys. Rev. B 70 (2004) 014113.

[27] E. J. O'Loughlin, S. D. Kelly, R. E. Cook, R. Csencsits, K. M. Kemner, Environ. Sci. \& Technol. 37 (2003) 721. 
D. Bocharov, M. Chollet, M. Krack, J. Bertsch, D. Grolimund, M. Martin, A. Kuzmin, J. Purans, E. Kotomin, Analysis of the $\mathrm{U}$ L3-edge $\mathrm{X}$-ray absorption spectra in $\mathrm{UO} 2$ using molecular dynamics simulations, Prog. Nucl. Energy 94 (2017) 187-193.

[28] N. T. Barrett, G. N. Greaves, B. T. M. Willis, G. M. Antonini, F. R. Thornley, J. Phys. C: Solid State Phys. 21 (1988) L791.

[29] S. Hubert, J. Purans, G. Heisbourg, P. Moisy, N. Dacheux, Inorg. Chem. 45 (2006) 3887-3894.

[30] S. D. Conradson, T. Durakiewicz, F. J. Espinosa-Faller, Y. Q. An, D. A. Andersson, A. R. Bishop, K. S. Boland, J. A. Bradley, D. D. Byler, D. L. Clark, D. R. Conradson, L. L. Conradson, A. L. Costello, N. J. Hess, G. H. Lander, A. Llobet, M. B. Martucci, J. Mustre de Leon, D. Nordlund, J. S. Lezama-Pacheco, T. E. Proffen, G. Rodriguez, D. E. Schwarz, G. T. Seidler, A. J. Taylor, S. A. Trugman, T. A. Tyson, J. A. Valdez, Phys. Rev. B 88 (2013) 115135.

[31] J. J. Rehr, J. J. Kas, F. D. Vila, M. P. Prange, K. Jorissen, Phys. Chem. Chem. Phys. 12 (2010) 5503-5513.

[32] A. Kuzmin, J. Purans, M. Benfatto, C. R. Natoli, Phys. Rev. B 47 (1993) 2480.

[33] A. Kuzmin, J. Purans, J. Phys.: Condens. Matter 5 (1993) 267.

Institute of Solid State Physics, University of Latvia as the Center of Excellence has received funding from the European Union's Horizon 2020 Framework Programme H2020-WIDESPREAD-01-2016-2017-TeamingPhase2 under grant agreement No. 739508, project CAMART² 